\title{
Neural mechanisms of three-dimensional vision
}

\author{
Ken-Ichiro Tsutsui ${ }^{\mathrm{a}, *}$, Masato Taira ${ }^{\mathrm{b}}$, Hideo Sakata ${ }^{\mathrm{b}, \mathrm{c}}$ \\ ${ }^{\mathrm{a}}$ Department of Anatomy, University of Cambridge, Downing Street, Cambridge CB2 3DY, UK \\ ${ }^{\mathrm{b}}$ Division of Applied System Neuroscience, Advanced Medical Research Center, Nihon University School of Medicine, \\ 30-1, Oyaguchi-kamimachi, Itabashi, Tokyo 173-0081, Japan \\ ${ }^{\mathrm{c}}$ Laboratory for Anatomy and Physiology, Tokyo Seiei College 1-4-6, Nishi-shinkoiwa, \\ Katsushika, Tokyo 124-8530, Japan
}

Received 21 July 2004; accepted 18 November 2004 Available online 18 January 2005

\begin{abstract}
We can see things in three dimensions because the visual system re-constructs the three-dimensional (3D) configurations of objects from their two-dimensional (2D) images projected onto the retinas. The purpose of this paper is to give an overview of the psychological background and recent physiological findings concerning three-dimensional vision. Psychophysical and computational studies have suggested that in the visual system the 3D surface orientation is first estimated independently from individual depth cues-such as binocular disparity, as well as various monocular cues including texture gradients - and then the information from these different depth cues is integrated to construct a generalized representation of the 3D surface geometry. Neurons involved in low-level disparity processing, or the detection of local absolute disparity, were found mainly in the occipital cortex, whereas neurons involved in high-level disparity processing, or the reconstruction of 3D surface orientation through the computation of disparity gradients, were found mainly in the parietal area caudal intraparietal sulcus (CIP). Neurons sensitive to texture gradients, which is one of the major monocular cues, were also found in CIP. The majority of these neurons were sensitive to disparity gradients as well, suggesting their involvement in the computation of 3D surface orientation. In CIP, neurons sensitive to multiple depth cues were widely distributed together with those sensitive to a specific depth cue, suggesting CIP's involvement in the integration of depth information from different sources. In addition, human and monkey imaging studies have indicated convergence of multiple depth cues in CIP. These neurophysiological findings suggest that CIP plays a critical role in 3D vision by constructing a generalized representation of the 3D surface geometry of objects.
\end{abstract}

(C) 2004 Elsevier Ireland Ltd and the Japan Neuroscience Society. All rights reserved.

Keywords: 3D vision; Area CIP; Binocular disparity; Texture gradient

\section{Introduction}

Seeing things in depth is not a simple task for the visual system. Spatial information is non-reversibly compressed and reduced, as the three-dimensional (3D) world is projected onto the two-dimensional (2D) surface of two retinas. For us to perceive depth, the visual system has to reconstruct $3 \mathrm{D}$ configurations of objects, their structures and locations in space, from their 2D image on the retinas. Clues for depth reconstruction, which are embedded in the 2D retinal image, are called 'depth cues'. How the visual system builds up a 3D representation of the visual world using these

\footnotetext{
* Corresponding author.

E-mail address: kit21@cam.ac.uk (K.I. Tsutsui).
}

depth cues has long been a major interest of vision research. Recently, there have been several important electrophysiological findings concerning the biological background of 3D vision in the brain, including our series of studies regarding area caudal intraparietal (CIP) in the parietal cortex. The objective of this paper is to give an overview of the neural mechanisms of 3D vision, with some emphasis on our recent findings in the parietal area CIP.

\section{Psychological background of $3 D$ vision}

Wheatstone (1838) was the first to demonstrate empirically that binocular disparity elicits the sense of depth. He used an instrument, called a 'stereoscope', of his own 
invention to stimulate the left and right eye independently. With that 'dichoptic' stimulation, he demonstrated that a slight difference in the positions and shapes of the corresponding images between the left and right eye produces a strong sensation of depth. In parallel to Wheatstone's empirical work, the basic geometry of binocular disparity was completed through theoretical works (Müller, 1826; Volkmann, 1836; Helmholtz, 1910) (Fig. 1). Another important step towards an understanding of the role of binocular disparity in depth vision was Julesz's (1964) invention of the random-dot stereogram (RDS). In a RDS, 3D shapes are defined solely by the disparities between the corresponding dots of the left and right eye image; therefore, they can be seen only after fusing the

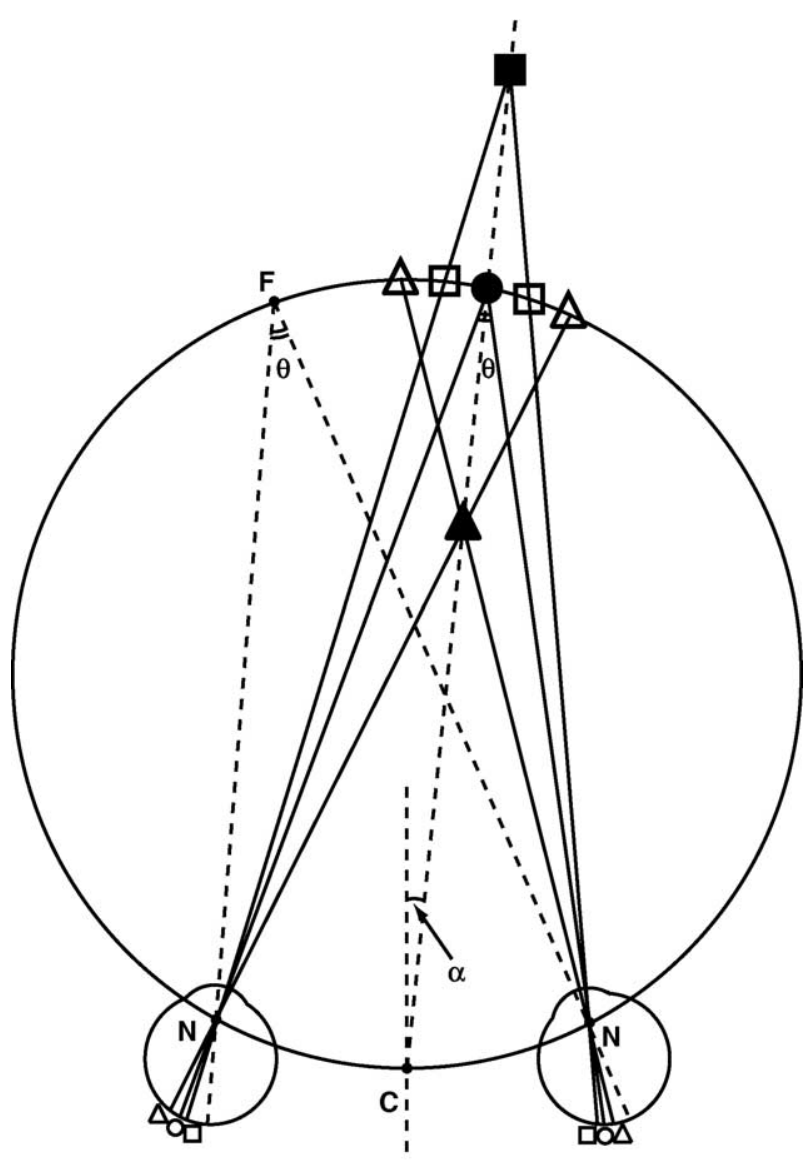

Fig. 1. Basic geometry of binocular disparity. A circle passing through the fixation point $(\mathrm{F})$ and nodal points $(\mathrm{N})$ of the eyes is called a horopter, or Vieth-Müller circle. An object anywhere on the horopter (large solid circle) produces zero disparity, having the same binocular subtense as the convergence angle for the fixation point $(\theta)$. An object inside the horopter (solid triangle), whose image can be projected to the horopter with convergence (large open triangles), produces crossed (near) disparity, or retinal images shifted to the temporal side from the retinal images of an object on the horopter in the same cyclopean azimuth (small open triangles). In contrast, an object outside the horopter (solid square), whose image can be projected to the horopter with no convergence (large open squares), produces uncrossed (far) disparity, or retinal images shifted to the nasal side from the retinal images of an object on the horopter in the same cyclopean azimuth (small open squares). C: cyclopean point; $\alpha$ : angle of cyclopean azimuth. images for both eyes. This clearly demonstrates the principles of stereoscopic vision that binocular disparity alone can elicit a strong 3D perception and that binocular disparity can be detected independently of 2D contour detection. The invention of the RDS had a strong impact on researchers at that time, many of whom believed that binocular disparity could be detected only after contour detection.

A psychophysical theory of vision by Gibson (1950a) was possibly the first attempt to explain scientifically why the perception of depth occurs with monocular cues, such as pictorial cues of texture, shading, and linear perspective, as well as motion. He suggested that the visual system extracts spatial information from a 2D retinal image by detecting 'gradients', increases or decreases of visual elements-e.g., size and density gradients in texture, luminance gradients in shading, speed gradients in motion. Following Gibson's work, many psychophysical studies proved that humans perceive depth with high precision from various monocular cues, such as texture gradients (Gibson, 1950b; Gruber and Clark, 1956), linear perspective (Clark et al., 1955; Olson, 1974; Stevens, 1983), and motion parallax (Braunstein, 1968).

Marr (1982) suggested in his computational theory of vision that a major goal of the visual system is to establish a representation of the 3D structure of an object in objectcentered coordinates. In his model, an important intermediate step towards the goal is to establish a viewercentered representation of the surface geometry of an object. He suggested that depth information may first be extracted independently from individual depth cues - such as binocular disparity, texture gradient, shading, and motion parallax - and then information from these different sources may be integrated to establish a generalized representation of the surface geometry. Such a model implies that the visual system is capable of extracting depth information from individual depth cues, and that there is interaction between the information from different depth cues at the perceptual level. As mentioned, there have been several psychophysical studies demonstrating the perception of depth from individual depth cues (Gibson, 1950b; Clark et al., 1955; Gruber and Clark, 1956; Braunstein, 1968; Olson, 1974; Stevens, 1983). Furthermore, interaction of monocular and binocular depth cues at the perceptual level has been demonstrated using the adaptation method: adaptations to texture gradients or motion parallax biased the perception of surface orientation based on binocular disparity (Poom and Borjesson, 1999).

\section{Neural correlates of $3 D$ vision}

\subsection{Loss of $3 D$ visual perception following a brain lesion}

An important case was reported by Holmes and Horrax (1919) concerning 3D vision and a brain lesion. It was about 

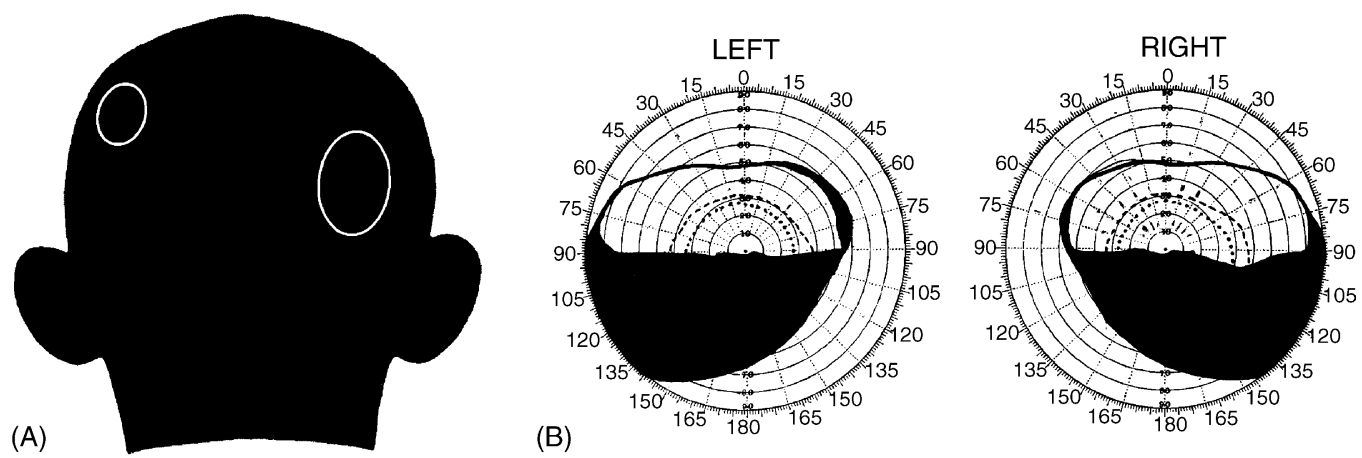

Fig. 2. Case W.F., in which a focal brain lesion resulted in a total loss of $3 \mathrm{D}$ visual perception. (A) Locations of entrance and exit wounds in posterior view of the patient's head. The center of brain lesion was estimated to be close to the angular gyrus in both hemispheres, judging from the position of the wounds on the skull. (B) Visual field of the patient measured within a perimeter. The field where the patient could not see a light spot is shown in black. Loss of the visual field was limited to the lower quadrants, which may suggest that the wound could have extended from the parietal to the occipital cortex, leaving the visual cortex ventral to the calcarine fissure intact. (From Holmes and Horrax (1919).)

a patient (W.F.) who completely lost 3D vision, with little impairment in 2D vision, after a gunshot caused a bilateral lesion in the parietal cortex centered around the angular gyrus (Fig. 2). His visual perception was completely altered after the injury so that every visual object lost its thickness and appeared flat to him. The symptom can be understood as an inability to perceive depth from any type of depth cue, including binocular disparity and all sorts of pictorial cues, rather than a mere loss of stereopsis (binocular vision); we know that a deliberate loss of stereopsis, which happens when we close one eye, does not result in a total loss of 3D perception. A more common symptom of parietal lesion is constructional apraxia. Patients show difficulty in assembling bricks according to a model pattern of bricks constructed three-dimensionally (Critchley, 1953). This symptom can be interpreted as impairment in perceiving the relationships of brick elements in 3D space, which would normally be achieved by using monocular and binocular depth cues.

We tried to experimentally replicate the impairment in $3 \mathrm{D}$ vision following a parietal lesion through reversible inactivation of the parietal area CIP with monkeys as subjects (Tsutsui et al., 2001). Monkeys were trained to perform a delayed-match-to-sample task in which they had to discriminate 3D surface orientations or 2D shapes. Following a micro-injection of muscimol, which reversibly blocks the function of neurons around injection as GABA agonist, into CIP, monkeys showed a selective impairment in 3D surface orientation discrimination while showing no change in 2D shape discrimination.

Clinical observations of the impairment of 3D vision following parietal lesions and their experimental replication using monkeys suggest that $3 \mathrm{D}$ vision is a discrete perceptual entity, and that the parietal cortex acts as a key structure for 3D visual perception. Furthermore, they suggest that the ventral visual pathway, which has been claimed to be most responsible for object vision (Ungerleider and Mishkin, 1982), cannot produce normal 3D visual perception without an intact parietal cortex.

\subsection{Disparity detection and representation of the $3 D$ surface orientation}

Disparity sensitive neurons in the brain were first found in cat visual cortex (Barlow et al., 1967; Nikara et al., 1968); later, similar neurons were found in monkey visual cortex such as V1, V2, V3, and V3A (Hubel and Livingstone, 1987; Poggio et al., 1985, 1988; Adams and Zeki, 2001). The neurons in these areas had relatively small receptive fields with retinotopic organization, and were tuned to a specific disparity ('near', 'far', or 'zero' disparity). The response of most disparity sensitive neurons in V1 was found to alter when the absolute disparity was changed, leaving the relative disparity constant (Cumming and Parker, 1999).

We found a group of disparity sensitive neurons in area CIP (caudal intraparietal) in the parietal cortex, which borders V3A and LIP at its posterior and anterior sides, respectively (Fig. 3) (Taira et al., 2000). CIP may be analogous to LOP, which was identified as being distinct from the adjacent V3A and LIP in the cytoarchitecture (Lewis and Van Essen, 2000). Neurons in CIP had relatively large receptive fields, ranging from $10^{\circ}$ to more than $30^{\circ}$ in diameter, sometimes covering more than half of the visual field, with no retinotopic organization. They responded selectively to the orientation of a flat surface that was defined by binocular disparity gradients in the random-dot stereogram and/or the solid-figure stereogram (SFS), suggesting that there are two different types of disparity processing. Neurons that show surface orientation selectivity for the RDS may compute the surface orientation from disparity gradients across the surface, whereas neurons that show surface orientation selectivity for the SFS may compute surface orientation from disparity gradients along the contours. We changed the absolute disparity while keeping the relative disparity unchanged by manipulating the depth position of the fixation spot (Fig. 4). When the fixation spot was placed in front of the surface, the whole surface fell into 'far' disparity, and when the fixation spot was placed behind the surface, the whole surface fell into 'near' disparity. 


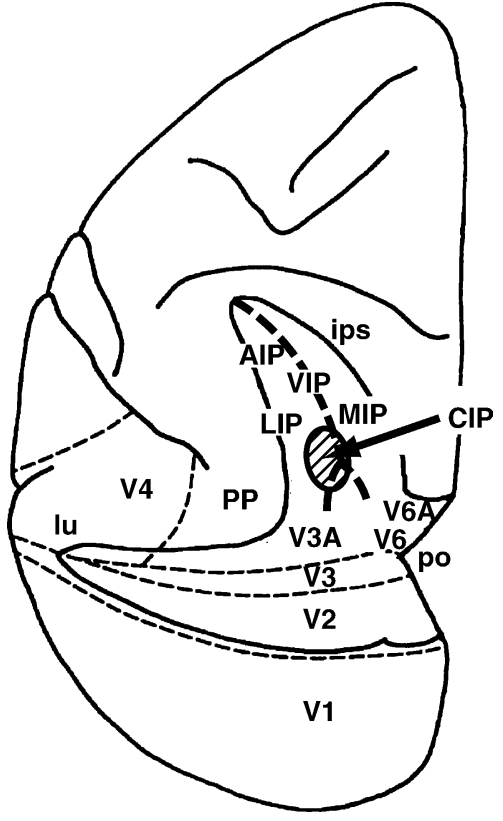

Fig. 3. Schematic illustration indicating the location of CIP in the top view of the left hemisphere. The intraparietal sulcus (ips), lunate sulcus (lu), and parietooccipital sulcus (po) are unfolded. AIP: anterior intraparietal; VIP: ventral intraparietal; MIP: medial intraparietal; LIP: lateral intraparietal; PP: posterior parietal. CIP is located caudally and laterally in the intraparietal sulcus, bordering LIP on the anterior side and V3A on the posterior side.

Regardless of this manipulation, disparity sensitive neurons in CIP responded to a specific surface orientation, suggesting that they are tuned to a specific disparity gradient, or relative disparity, independently of the absolute disparity.
The above findings may demonstrate an important difference in the properties of the disparity sensitive neurons in CIP compared with those in the occipital cortex: while neurons in the occipital cortex are involved in low-level disparity detection within their relatively small receptive fields, CIP neurons may be involved in high-level computation of the $3 \mathrm{D}$ orientation of a relatively wide surface, based on disparity information that comes up from the occipital cortex. In accordance with this idea, in a monkey f-MRI study CIP showed increased activity accompanying an increase in the complexity of disparitydefined patterns, while the early visual cortices did not (Tsao et al., 2003).

There are some other important findings that demonstrate the difference of disparity coding between the early visual cortices and CIP. In an anti-correlated RDS (acRDS), the contrast polarity of the corresponding dots between the left and right eyes is inverted, so that a black dot in the left eye corresponds to a white dot in the right eye. The acRDS normally does not cause any depth perception. It was found that V1 neurons are sensitive to the acRDS and show inverted disparity tuning (Cumming and Parker, 1997), suggesting that these neurons are involved in low-level disparity detection at a sub-perceptual level. In contrast, we found that most CIP neurons show little response to the acRDS (unpublished data), which suggests that CIP neuron activity can be directly related to perception.

Recent studies of occipital disparity sensitive neurons suggest that binocular disparity signals may be progressively processed in a bottom-up manner through the occipital cortex in order to extract relative disparity from absolute disparity. Almost all neurons in V1 were found to be

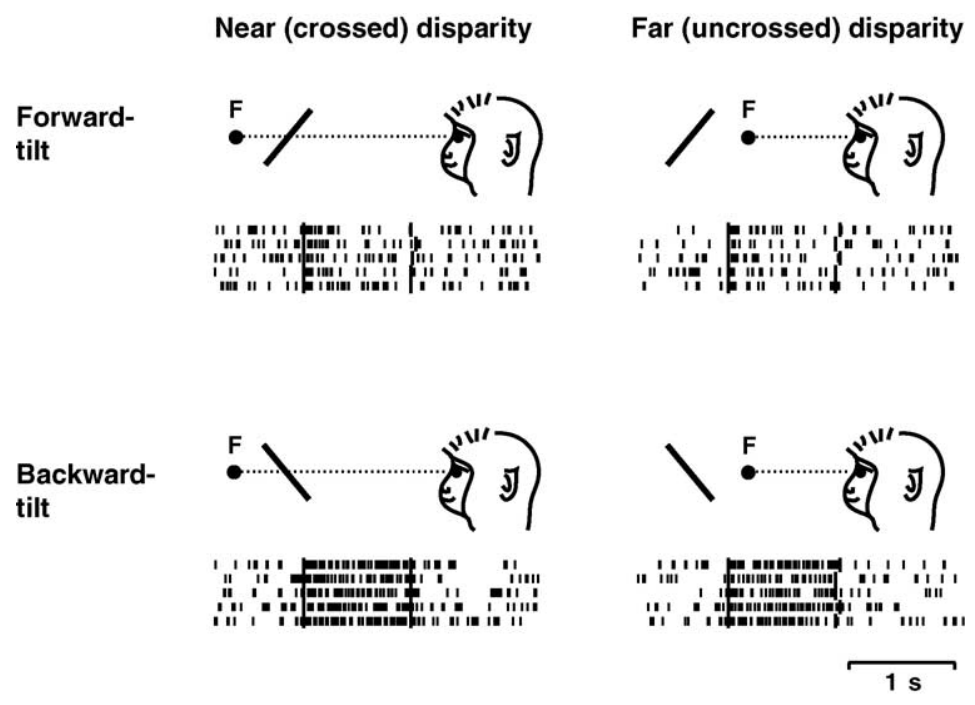

Fig. 4. Activities of a typical CIP neuron sensitive to a 3D surface orientation defined by disparity gradients. Figures above the rasters schematically indicate the stimulus orientation and the position of the fixation spot. Rasters were aligned at the onset of the stimulus (vertical lines), and markers indicate the time of stimulus offset. The neuron response was tested for five different surface orientations (only two are shown) using random-dot stereograms (RDSs): top row, forward-tilt; bottom row, backward-tilt. Keeping the viewing distance of the stimulus constant, the fixation spot (F) was placed behind (left column) or in front of (right column) the stimulus, so that any point of the stimulus surface would have crossed or uncrossed disparity, respectively. Note that this neuron was responding to the backward-tilt, regardless of the difference of the absolute disparity (crossed/uncrossed). 
sensitive to absolute disparity alone (Cumming and Parker, 1999). In contrast, the majority of V2 neurons was found to have some sensitivity to relative disparity, although the influence of absolute disparity was still stronger in most of them (Thomas et al., 2002). In V3 and V3A, neurons were found to be in a columnar organization based on their selectivity to binocular disparity (Adams, 1997; Adams and Zeki, 2001), which may suggest that these areas are specialized for binocular disparity processing, such as spatial integration of local disparity and the computation of disparity gradient over a wide area. It was demonstrated that CIP receives direct fiber projections from V3 and V3A (Adams, 1997), which seem to be a major source of binocular disparity information for CIP. Neurons in CIP have relatively large receptive fields, sometimes covering more than half of the visual field, and show selectivity to a pure relative disparity, or disparity gradient, completely independent of absolute disparity.

In addition to our finding of disparity sensitive neurons in CIP, recent studies of the ventral visual pathway have reported disparity sensitive neurons, in cortical areas such as V4 (Hinkle and Connor, 2002; Watanabe et al., 2002) and the inferotemporal (IT) cortex (Janssen et al., 1999, 2000; Uka et al., 2000; Tanaka et al., 2001). Several findings suggest high-level disparity coding in these areas. Hinkle and Connor (2002) found that neurons in V4 respond selectively to a specific 3D orientation of a bar by combining 2D frontoparallel orientation tuning with relative disparity tuning. These neurons do not change 3D orientation tuning with the change of the absolute disparity. Janssen et al. (1999,
2000) found that neurons in the rostral part of the lower bank of the STS (area TEs) are sensitive to 3D curvature defined by a disparity gradient, and that these neurons do not change their selectivity to the curvature with a change of absolute disparity. Furthermore, it was found that disparity sensitive neurons in V4 (Tanabe et al., 2004) and TEs (Janssen et al., 2003) are not sensitive to the acRDS. Thus, neurons that appear to be involved in high-level disparity coding are found both in the dorsal and ventral pathways.

\subsection{Computation of the $3 D$ surface orientation based on monocular cues}

During the course of our experiment examining the properties of disparity sensitive neurons in CIP, we found that some of the disparity sensitive neurons maintain their selectivity to a $3 \mathrm{D}$ surface orientation in the absence of disparity cues if linear perspective cues are available (Tsutsui et al., 2001). This finding led us to undertake a systematic study of the effect of monocular cues on the response of CIP neurons using texture gradients (Fig. 5) (Tsutsui et al., 2002), since texture gradients may be one of the most powerful monocular cues as suggested by Gibson (1950a,b). Using a dot-texture pattern as a probe, we found a group of neurons that responded selectively to texture gradients. Of the neurons further examined with another texture pattern, a line-texture, the majority showed the same selectivity for texture gradients over two texture patterns (Fig. 6). Of the neurons further examined with texture elements of different sizes and densities, the majority

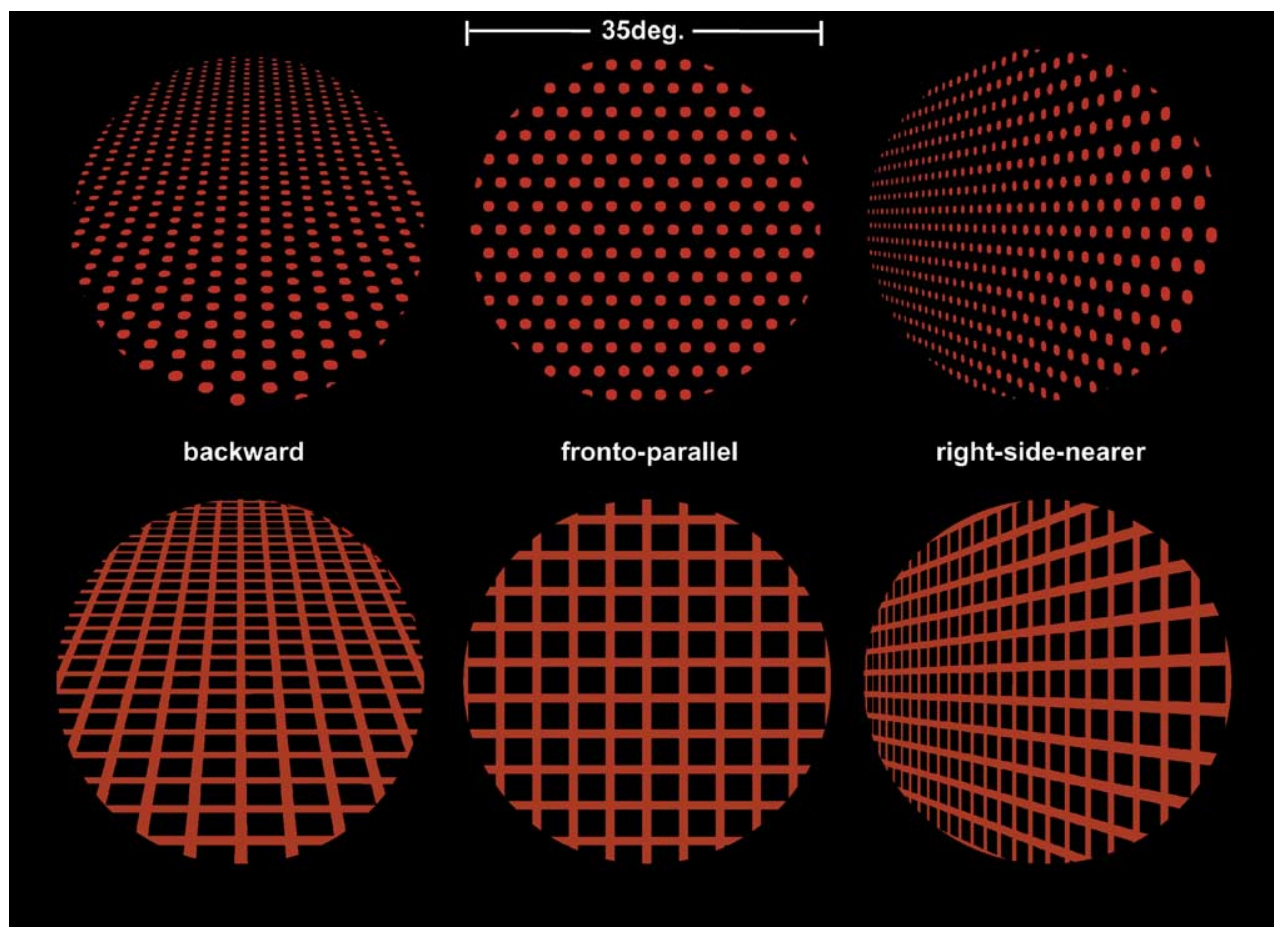

Fig. 5. Texture gradients and 3D surface orientation. Top row, dot-texture; bottom row, line-texture. These stimuli were used in Tsutsui et al. (2002). 
(A) Dot-TP
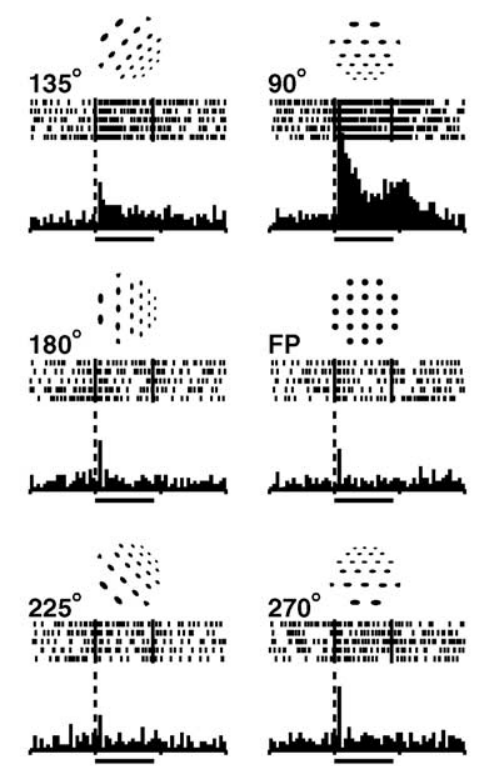
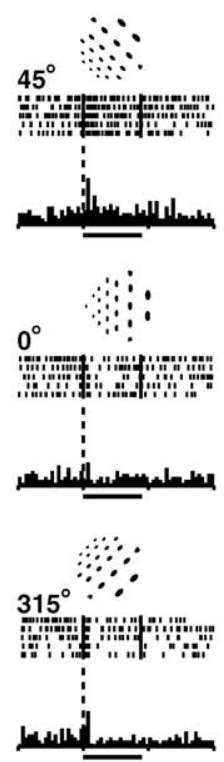

(B) Line-TP
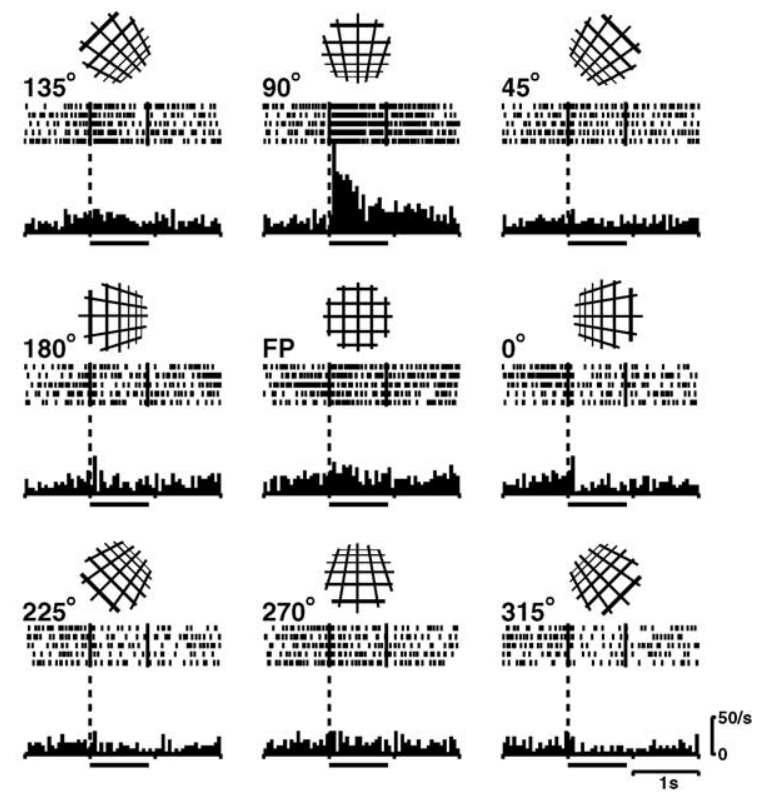

Fig. 6. Activities of a typical CIP neuron sensitive to texture gradients. The neuron response was tested for nine different orientations using dot- (A) and linetexture (B) patterns. Numbers in the top-left corner of the rasters represent the tilt angle. (FP indicates frontoparallel orientation.) This neuron was tuned to forward $\left(90^{\circ}\right)$ tilt, in which the top edge of the surface was nearer to the observer, regardless of the difference of texture pattern. (Adapted from Tsutsui et al. (2002).)

showed the same selectivity for texture gradients over the size and density changes of texture elements (unpublished data). These findings suggest that the neurons in CIP extract the texture gradient information from the arbitrary pattern, size, and density of a texture, to establish a representation of the 3D surface orientation. Very recently, Liu et al. (2004) found similar neurons that are sensitive to a disparity gradient, in the rostral part of the lower bank of the STS (TEs).

Sugihara et al. (2002) studied the depth coding based on another type of monocular cues. They found a group of neurons in MSTd that seem to represent the 3D surface orientation based on motion gradients. These neurons showed selectivity to a 3D surface orientation defined by rotating dynamic random dots, with high response invariance over different stimulus positions within their receptive fields. As it was reported that selectivity to motion parallax can be found in the level of MT (Treue and Andersen, 1996; Xiao et al., 1997), it appears that MSTd neurons are integrating motion parallax signals coming up from MT, in order to code the 3D surface orientation based on motion gradients independently of the local motion directions and speeds. Having motion sensitivity as a primary feature, neurons in MT (Maunsell and Van Essen, 1983; DeAngelis and Newsome, 1999; Naguyenkim and DeAngelis, 2003) and MST (Roy et al., 1992; Sakata et al., 1994) were found to show selectivity to binocular disparity as well, which may facilitate the computation of depth from motion by reducing the ambiguity in the stimulus.

\subsection{Integrated representation of the $3 D$ surface orientation}

Marr (1982) suggested that a generalized representation of the 3D surface geometry might be realized by integrating information from different sources following the extraction of depth information from individual depth cues. Consistent with his prediction, we found a group of neurons that are selective to the $3 \mathrm{D}$ surface orientation and sensitive to multiple depth cues (Tsutsui et al., 2001, 2002). Of the texture gradient sensitive neurons found in CIP, 77\% also showed selectivity to a 3D surface orientation defined by RDS disparity gradients. The most interesting finding was that the tuning for surfaces defined by texture gradients is almost identical to that for surfaces defined by disparity gradients in the neurons sensitive to both disparity and texture gradients (Fig. 7). Such convergence of coherent information from different sources is very unlikely to happen by chance; therefore, it strongly suggests that these neurons are involved in the perception of the 3D surface orientation. Through the integration of disparity and texture gradient signals, CIP neurons seem to construct a generalized representation of the $3 \mathrm{D}$ surface orientation. Following our study, Liu et al. (2004) found a similar convergence effect for the disparity and texture gradient signals in texture-gradient-sensitive neurons in the rostral part of the lower bank of the STS (TEs).

The concentrated distribution of neurons integrating information from multiple depth cues suggests that the brain region is a key structure for $3 \mathrm{D}$ vision. Recent functional imaging studies of monkeys and humans also suggest the 

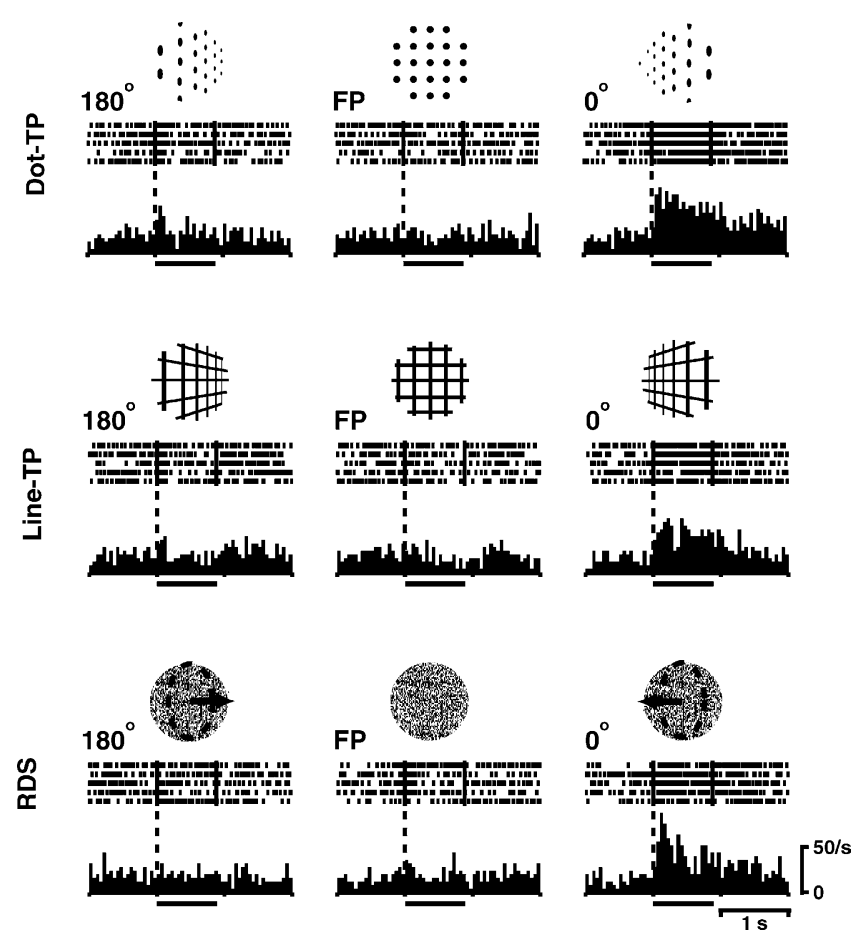

Fig. 7. Activities of a typical CIP neuron sensitive to both disparity and texture gradients. The neuron response was tested for nine different orientations (only three are shown) using dot- and line-texture patterns, as well as random-dot stereograms (RDSs). Note that the preferred orientation (right side edge being nearer to the observer, $0^{\circ}$ tilt) was the same regardless of the difference of the depth cue (texture gradients in the dotand line-texture patterns, and disparity gradients in the RDSs). (Adapted from Tsutsui et al. (2002).)

convergence of multiple depth cues in CIP. In monkey f-MRI studies, it has been reported that CIP is activated when monkeys view disparity-defined mosaics in RDSs (Tsao et al., 2003), and when they are shown 3D shapes defined by texture gradients or motion parallax (Sereno et al., 2002). In humans, the caudal intraparietal area, a human homologue of CIP, is activated when subjects view disparity-defined mosaics (Tsao et al., 2003) or discriminating disparitydefined 3D surface orientation (Naganuma et al., 2004) in RDSs, and when they attend to a 3D visual feature defined by texture gradients (Shikata et al., 2001) or shading (Taira et al., 2001).

As we can find neurons responding to a single depth cue together with neurons responding to multiple cues in CIP, it may be that depth signals from an individual depth cue, which are independently processed through the occipital cortex, are projected in parallel to CIP, and then integrated by multiple cue neurons. Binocular disparity signals may be processed mainly through the dorsal visual pathway before reaching CIP. Neurons sensitive to binocular disparity are found in the monkey V1, V2, V3, and V3A (Hubel and Livingstone, 1987; Poggio et al., 1985, 1988; Adams, 1997; Adams and Zeki, 2001). Particularly in V3 and V3A, neurons were found to be in a columnar organization based on their selectivity to binocular disparity (Adams, 1997;
Adams and Zeki, 2001), which may suggest that these areas are specialized for binocular disparity processing. CIP was found to receive fiber projections from V3 and V3A (Adams, 1997), and therefore it appears to receive binocular disparity information that has been processed through the occipital cortex in a bottom-up manner. In contrast to binocular disparity cues, we have relatively little knowledge about the neural mechanisms of depth-information processing concerning monocular cues. Little is known about the neural process of extracting depth information from texture gradients before the level of CIP. With regard to depthfrom-motion, neurons that appear to code the 3D surface orientation by integrating motion gradients were found in MSTd (Sugihara et al., 2002). However, we still lack evidence that CIP receives information from MSTd, or that CIP neurons are sensitive to motion gradients.

\section{Summary and future prospects for studies of 3D vision}

The purpose of this paper was to provide an overview of the neural mechanisms of 3D vision by reviewing relevant psychological and physiological studies. The findings of these studies can be summarized as follows. (1) Psychophysical and computational studies suggest that a 3D surface orientation can be estimated independently in the visual system from individual depth cues, such as binocular disparity, linear perspective, texture gradient, and motion parallax, and the information derived from these different depth cues is integrated to create a generalized representation of the 3D surface geometry. (2) A clinical neuropsychology study (case W.F.) shows that a severe impairment of 3D visual perception can result from a bilateral parietal cortex lesion. A similar effect was observed after reversible inactivation of parietal area CIP in monkeys. (3) Neurons involved in low-level disparity processing, or the detection of local absolute disparity, can be found mainly in the occipital cortex, whereas neurons involved in high-level disparity processing, or the reconstruction of a 3D surface orientation through the computation of a disparity gradient, can be found mainly in the parietal area CIP and the inferotemporal area TEs. (4) Neurons sensitive to texture gradients, which provide a major monocular cue, can be found in CIP and TEs. The majority of these neurons are sensitive to disparity gradients as well, suggesting their involvement in the computation of 3D surface orientation. (5) In CIP, neurons sensitive to multiple depth cues are intermingled with those sensitive to a specific depth cue, suggesting CIP's involvement in the integration of depth information from different sources. Human and monkey imaging studies also suggest the convergence of multiple depth cues in CIP.

As summarized above, the basic framework of 3D visual processing in the brain proposed by Marr, in which depth information is first extracted independently from individual 
depth cues, and then the information from these different sources is integrated to establish a generalized representation of the surface geometry, has now obtained biological validity. However, much remains to be studied, as follows. (1) It has been demonstrated that information of multiple depth cues, such as binocular disparity and monocular cues of texture gradients and linear perspective, converge in CIP. However, sensitivity of CIP neurons to some other important monocular cues, such as motion parallax and shading, has yet to be investigated. (2) We do not have enough neuronal data yet to build up a detailed model that can explain how depth and 3D surface orientation are processed through occipital cortex in a bottom-up manner before it reaches CIP. In order to examine the details of the bottom-up processing of binocular disparity signals, properties of disparity sensitive neurons in different areas have to be systematically investigated by manipulating relative disparity and disparity gradient using identical stimuli. We have even less knowledge about how depth and 3D surface orientation are computed from texture gradients, linear perspective, and other monocular cues in the occipital cortex. It is necessary to examine how occipital neurons respond to depth information in these monocular cues. (3) The differences between the two brain areas that appear to be deeply involved in 3D visual perception, CIP in the parietal cortex and TEs in the inferotemporal cortex, have to be investigated. Cases in clinical and experimental neuropsychology suggest that the parietal cortex is indispensable for $3 \mathrm{D}$ visual perception, and that $3 \mathrm{D}$ visual perception cannot be achieved through only the ventral visual pathway, which includes TEs. That does not necessarily mean that CIP is more important than TEs; however, we need to examine whether TEs inactivation causes the same impairment as CIP inactivation. Neuronal recording and reversible inactivation of both areas using the same visual stimuli and behavioral tasks may be an effective way to investigate how they differ and how they cooperate in $3 \mathrm{D}$ visual perception. (4) Providing that the purpose of the visual system is to recognize complex 3D structures of objects, the process beyond the representation of a 3D surface geometry is not yet known. In the anterior intraparietal sulcus area (AIP) of monkeys trained to manipulate $3 \mathrm{D}$ objects, some neurons have been found to respond selectively to 3D objects even when the monkeys are required to look at the object without any hand movement (Murata et al., 1996, 2000). These findings suggest that within the parietal cortex, the 3D structures of objects may be computed based on the 3D surface geometry, while the information proceeds from CIP to AIP through the other sub-regions of the parietal cortex. It may be important to systematically examine the response properties of parietal neurons according to 3D object structures, using models proposed for the description of basic 3D shape recognition such as 'shape index' and 'curvedness' by Koenderink (1990) or 'geons' by Biederman (1990). (5) Neural mechanisms beyond the stimulus driven, bottom-up perception of a $3 \mathrm{D}$ object, which may involve short-term memory and mental manipulation of 3D objects, have yet to be examined. For instance, to recognize the structure of a large object, one has to actively scan the surface of the object by moving the eyes, and then construct a representation of the entire object by putting together the information sequentially obtained. Our recent finding that changes in CIP neuron activity is related to short-term memory and same/different discrimination of 3D surface orientation (Tsutsui et al., 2003) suggests that the parietal cortex may be involved in such a cognitive process concerning $3 \mathrm{D}$ vision.

\section{Acknowledgements}

This study was supported by: a grant from the Ministry of Education, Culture, Sports, Science, and Technology to promote multi-disciplinary research projects "Brain mechanisms for Cognition, Memory and Behavior" at Nihon University; a grant-in-aid for scientific research on priority areas-advanced brain science project-from the Ministry of Education, Culture, Sports, Science, and Technology (\#16015303); a grant for basic sciences from the Sumitomo Foundation; and a grant from Satow's Research Fund for Behavioral Science.

\section{References}

Adams, D.L., 1997. Functional organization of the monkey visual cortex for stereoscopic depth. Ph.D. dissertation. University College London, London.

Adams, D.L., Zeki, S., 2001. Functional organization of macaque V3 for stereoscopic depth. J. Neurophysiol. 86, 2195-2203.

Barlow, H.B., Blakemore, C., Pettigrew, J.D., 1967. The neural mechanism of binocular depth discrimination. J. Physiol. Lond. 193, 327-342.

Biederman, I., 1990. Higher-vision. In: Osherstein, D.N., Kosslyn, M., Hollerbach, J.M. (Eds.), Visual Cognition and Action 2, MIT Press, Cambridge, pp. 41-72.

Braunstein, M.L., 1968. Motion and texture as sources of slant information. J. Exp. Psychol. 78, 247-253.

Clark, W.C., Smith, A.H., Rabe, A., 1955. Retinal gradient of outline as a stimulus for slant. Can. J. Psychol. 9, 247-253.

Critchley, 1953. The Parietal Lobes. Edward Arnold, London.

Cumming, B.G., Parker, A.J., 1997. Responses of primary visual cortical neurons to binocular disparity without depth perception. Nature 389, 280-283.

Cumming, B.G., Parker, A.J., 1999. Binocular neurons in V1 of awake monkeys are selective for absolute, not relative, disparity. J. Neurosci. 19, 5602-5618.

DeAngelis, G.C., Newsome, W.T., 1999. Organization of disparity-selective neurons in macaque area MT. J. Neurosci. 19, 1398-1415.

Gibson, J.J., 1950a. The Perception of the Visual World. Houghton Mifflin, Boston.

Gibson, J.J., 1950b. The perception of visual surfaces. Am. J. Psychol. 63, 367-384.

Gruber, H.E., Clark, W.C., 1956. Perception of slanted surfaces. Percept. Mot. Skills 6, 97-106.

Helmholtz, H. von, 1910. Handbuch der Physiologischen Optik. Voss, Hamburg.

Hinkle, D.A., Connor, C.E., 2002. Three-dimensional orientation tuning in macaque area V4. Nat. Neurosci. 5, 665-670. 
Holmes, G., Horrax, G., 1919. Disturbances of spatial orientation and visual attention with loss of stereoscopic vision. Arch. Neurol. Psychiatry 1, 385-407.

Hubel, D.H., Livingstone, M.S., 1987. Segregation of form, color, and stereopsis in primate area 18. J. Neurosci. 7, 3378-3415.

Janssen, P., Vogels, R., Orban, G.A., 1999. Macaque inferior temporal neurons are selective for disparity-defined three-dimensional shapes. Proc. Natl. Acad. Sci. U.S.A. 96, 8217-8222.

Janssen, P., Vogels, R., Orban, G.A., 2000. Three-dimensional shape coding in inferior temporal cortex. Neuron 27, 385-397.

Janssen, P., Vogels, R., Liu, Y., Orban, G.A., 2003. At least at the level of inferior temporal cortex, the stereo correspondence problem is solved. Neuron 37, 693-701.

Julesz, B., 1964. Binocular depth perception without familiarity cues. Science 145, 356-362.

Koenderink, J.J., 1990. Solid Shape. MIT Press, Cambridge.

Lewis, J.W., Van Essen, D.C., 2000. Mapping of architectonic subdivisions in the macaque monkey, with emphasis on parieto-occipital cortex. J. Comp. Neurol. 428, 79-111.

Liu, Y., Vogels, R., Orban, G.A., 2004. Convergence of depth from texture and depth from disparity in macaque inferior temporal cortex. J. Neurosci. 24, 3795-3800.

Marr, D., 1982. Vision. Freeman, San Francisco.

Maunsell, J.H., Van Essen, D.C., 1983. Functional properties of neurons in middle temporal visual area of the macaque monkey. II. Binocular interactions and sensitivity to binocular disparity. J. Neurophysiol. 49, 1148-1167.

Müller, J., 1826. Zur Vergleichenden Physiologie des Gesichtssinnes des Menschen und der Thiere. Cnobloch, Leibzig.

Murata, A., Gallese, V., Luppino, G., Kaseda, M., Sakata, H., 2000. Selectivity for the shape, size, and orientation of objects for grasping in neurons of monkey parietal area AIP. J. Neurophysiol. 83, 2580 2601.

Murata, A., Gallese, V., Kaseda, M., Sakata, H., 1996. Parietal neurons related to memory-guided hand manipulation. J. Neurophysiol. 75, 2180-2186.

Naganuma, T., Nose, I., Inoue, K., Takemoto, A., Katsuyama, N., Taira, M., in press. Information processing of geometrical features of a surface based on a binocular disparity cues: an fMRI study. Neurosci. Res. in press.

Naguyenkim, J.D., DeAngelis, G.C., 2003. Disparity-based coding of threedimensional surface orientation by macaque middle temporal neurons. J. Neurosci. 23, 7117-7128.

Nikara, T., Bishop, P.O., Pettigrew, J.D., 1968. Analysis of retinal correspondence by studying receptive fields of binocular single units in cat striate cortex. Exp. Brain Res. 6, 353-372.

Olson, R.K., 1974. Slant judgments from static and rotating trapezoids correspond to rules of perspective geometry. Percept. Psychophys. 15, 509-516.

Poggio, G.F., Gonzalez, F., Krause, F., 1988. Stereoscopic mechanisms in monkey visual cortex: binocular correlation and disparity selectivity. J. Neurosci. 8, 4531-4550.

Poggio, G.F., Motter, B.C., Squatrito, S., Trotter, Y., 1985. Responses of neurons in visual cortex (V1 and V2) of the alert macaque to dynamic random-dot stereograms. Vis. Res. 25, 397-406.

Poom, L., Borjesson, E., 1999. Perceptual depth synthesis in the visual system as revealed by selective adaptation. J. Exp. Psychol. Hum. Percept. Perform. 25, 504-517.

Roy, J.P., Komatsu, H., Wurtz, R.H., 1992. Disparity sensitivity of neurons in monkey extrastriate area MST. J. Neurosci. 12, 2478-2492.

Sakata, H., Shibutani, H., Ito, Y., Tsurugai, K., Mine, S., Kusunoki, M., 1994. Functional properties of rotation-sensitive neurons in the posterior parietal association cortex of the monkey. Exp. Brain Res. 101, 183202.

Sereno, M.E., Trinath, T., Augath, M., Logothetis, N.K., 2002. Threedimensional shape representation in monkey cortex. Neuron. 33, 635652 .

Shikata, E., Hamzei, F., Glauche, V., Knab, R., Dettmers, C., Weiller, C., Buchel, C., 2001. Surface orientation discrimination activates caudal and anterior intraparietal sulcus in humans: an event-related fMRI study. J. Neurophysiol. 85, 1309-1314.

Stevens, K.A., 1983. Surface tilt (the direction of slant): a neglected psychophysical variable. Percept. Psychophys. 33, 241-250.

Sugihara, H., Murakami, I., Shenoy, K.V., Andersen, R.A., Komatsu, H., 2002. Response of MSTd neurons to simulated 3D orientation of rotating planes. J. Neurophysiol. 87, 273-285.

Taira, M., Tsutsui, K.I., Jiang, M., Yara, K., Sakata, H., 2000. Parietal neurons represent surface orientation from the gradient of binocular disparity. J. Neurophysiol. 83, 3140-3146.

Taira, M., Nose, I., Inoue, K., Tsutsui, K.I., 2001. Cortical areas related to attention to 3D surface structures based on shading: an fMRI study. Neuroimage 14, 959-966.

Tanabe, S., Umeda, K., Fujita, I., 2004. Rejection of false matches for binocular correspondence in macaque visual cortical area V4. J. Neurosci. 24, 8170-8180.

Tanaka, H., Uka, T., Yoshiyama, K., Kato, M., Fujita, I., 2001. Processing of shape defined by disparity in monkey inferior temporal cortex. J. Neurophysiol. 85, 735-744.

Thomas, O.M., Cumming, B.G., Parker, A.J., 2002. A specialization for relative disparity in V2. Nat. Neurosci. 5, 472-478.

Treue, S., Andersen, R.A., 1996. Neural responses to velocity gradients in macaque cortical area MT. Vis. Neurosci. 13, 797-804.

Tsao, D.Y., Vanduffel, W., Sasaki, Y., Fize, D., Knutsen, T.A., Mandeville, J.B., Wald, L.L., Dale, A.M., Rosen, B.R., Van Essen, D.C., Livingstone, M.S., Orban, G.A., Tootell, R.B., 2003. Stereopsis activates V3A and caudal intraparietal areas in macaques and humans. Neuron 39, $555-568$.

Tsutsui, K.I., Jiang, M., Yara, K., Sakata, H., Taira, M., 2001. Integration of perspective and disparity cues in surface-orientation-selective neurons of area CIP. J. Neurophysiol. 86, 2856-2867.

Tsutsui, K.I., Sakata, H., Naganuma, T., Taira, M., 2002. Neural correlates for perception of 3D surface orientation from texture gradient. Science 298, 409-412.

Tsutsui, K.I., Jiang, M., Sakata, H., Taira, M., 2003. Short-term memory and perceptual decision for three-dimensional visual features in the caudal intraparietal sulcus (Area CIP). J. Neurosci. 23, 5486-5495.

Uka, T., Tanaka, H., Yoshiyama, K., Kato, M., Fujita, I., 2000. Disparity selectivity of neurons in monkey inferior temporal cortex. J. Neurophysiol. 84, 120-132.

Ungerleider, L.G., Mishkin, M., 1982. Two cortical visual systems. In: Ingle, D.J., Mansfield, M.S., Goodale, M.S. (Eds.), The Analysis of Visual Behavior, MIT Press, Cambridge, pp. 549-586.

Volkmann, A.W., 1836. Neue Beiträge zur Physiologie des Gesichtssinnes. Breitkopft, Leipzig.

Watanabe, M., Tanaka, H., Uka, T., Fujita, I., 2002. Disparity-selective neurons in area V4 of macaque monkeys. J. Neurophysiol. 87, 19601973.

Wheatstone, C., 1838. Contributions to the physiology of vision-part the first. On some remarkable and hitherto unobserved phenomena of binocular vision. Philos. Trans. R. Soc. 128, 371-394.

Xiao, D.K., Marcar, V.L., Raiguel, S.E., Orban, G.A., 1997. Selectivity of macaque MT/V5 neurons for orientation in depth specified by motion. Eur. J. Neurosci. 9, 956-964. 